\title{
EMERGÊNCIA DE PLÂNTULAS DE Copernicia Hospita Martius EM FUNÇÃO DO TAMANHO DA SEMENTE, DO SUBSTRATO E AMBIENTE ${ }^{1}$
}

\author{
ALEXANDRE BOSCO DE OLIVEIRA², SEBASTIÃO MEDEIROS FILHO ${ }^{3}$, ANTÔNIO MARCOS ESMERALDO BEZERRA ${ }^{4}$, \\ RISELANE DE LUCENA ALCÂNTARA BRUNO ${ }^{5}$.
}

\begin{abstract}
RESUMO - A germinação lenta e desuniforme das sementes de palmeiras acarreta problemas na propagação das espécies pertencentes a esta família, dificultando, principalmente, a produção de mudas em escala comercial. Esse trabalho objetivou determinar o efeito do tamanho da semente, de substratos e do ambiente na emergência de plântulas de Copernicia hospita Martius. As sementes foram submetidas aos tratamentos: substratos [areia vermelha + bagana de carnaúba + húmus (2,5:2,5:1 em volume) e solo + arisco + composto orgânico Polefértil ${ }^{\circledR}$ (2:2:1 em volume)]; ambientes (pleno sol e casa de vegetação); e tamanho de sementes (pequena, média, grande e mistura). Os tratamentos foram comparados quanto à percentagem de emergência, avaliada aos 60 dias após a semeadura, índice de velocidade e tempo médio de emergência. Os fatores analisados não influenciam no percentual de emergência de plântulas. Sementes de C. hospita oriundas da mistura e de tamanho pequeno, semeadas em ambos os substratos analisados, sob casa de vegetação, proporcionam emergência mais rápida das plântulas.
\end{abstract}

Termos para indexação: carnaúba hospedeira, biometria da semente, meio de cultivo, sombreamento.

\section{EMERGENCY OF COPERNICIA HOSPITA MARTIUS SEEDLINGS DEPENDING ON THE SIZE SEED, OF THE SUBSTRATE AND ENVIRONMENT}

\begin{abstract}
The slow and desuniforme germination seeds of palm trees brings problems in the propagation of the species belonging to this family, more difficult, primarily, the production of seedlings on a commercial scale. This work objective was to determine the effect of the size seed, substrates and the environment in emergency of Copernicia hospita Martius seedlings. Seeds were referred to treatments: substrates [(red sand + carnauba straw + humus $(2,5: 2,5: 1$ by volume) and soil + dark sand + organic compound Polefértil ${ }^{\circledR}(2: 2: 1$ by volume)]; environments (sun and house of vegetation), and size seed (small, medium, large and mix). The treatments were compared in terms of emergency percentage, estimated to 60 days after sowing, speed emergency index and mean emergency time. The factors examined not affect the emergency percentage seedlings. Seeds of $C$.
\end{abstract}

${ }^{1}$ Submetido em 02/02/2008. Aceito para publicação em 19/08/2008. Parte da Dissertação de mestrado do primeiro autor.

${ }^{2}$ Eng. Agr., MSc., doutorando em Agronomia/Fitotecnia, CCA/UFC, bolsista do CNPq, Fortaleza-CE, aleufc@gmail.com.

${ }^{3}$ Eng. Agr., Prof. Dr., Depto de Fitotecnia, CCA/UFC, Caixa Postal 12.168,
60356-001, Fortaleza-CE, bolsista do CNPq, filho@ufc.br.

${ }^{4}$ Eng. Agr., Prof. Dr., Depto de Fitotecnia, CCA/UFC, Caixa Postal 12.168, 60356-001, Fortaleza-CE, esmeraldo@ufc.br.

${ }^{5}$ Eng. Agr., Prof. Dra., Depto de Fitotecnia, CCA/UFPB, Caixa Postal 02, 58397-000, Areia-PB, bolsista do CNPq, lane@cca.ufpb.br. 
hospita mixing and small size, sown in both substrates, under house of vegetation, provide emergency

fastest of the seedlings.

Index terms: hostess carnauba, seed biometry, culture medium, shade.

\section{INTRODUÇÃO}

A família Palmae ou Arecaceae compreende um grupo de plantas de grande importância econômica e ornamental, muito utilizadas em regiões tropicais, fornecendo ao homem, cocos, tâmaras, palmito, açúcar, sagu, óleo, cera, fibras e material para a construção de habitações rústicas, como folhas e estipe (Lorenzi et al., 2004).

A Copernicia hospita Martius, conhecida popularmente como carnaúba hospedeira (Lorenzi et al., 2004), é uma palmeira de tamanho médio, nativa das savanas e florestas secas da Cuba, podendo ser encontrada em montes e em inclinações. A parte específica do nome, hospita, é do latim, significa hospitalidade, devido ao fato desta árvore ser um repouso hospitaleiro para uma larga variedade de pássaros. Seu tronco colunar liso que pode crescer até 0,3 m no diâmetro e até 7,9 $\mathrm{m}$ de altura. As flores são hermafroditas, podendo, portanto, produzir sementes por meio de autopolinização. Até 40 folhas dão forma a um esboço circular muito característico em torno do alto do tronco (Broschat e Meerow, 2000).

A propagação das palmeiras se dá principalmente por sementes, as quais apresentam limitações como germinação lenta, irregular e freqüentemente em baixa porcentagem, para a maioria das espécies (Broschat, 1994).

A influência do tamanho das sementes sobre a qualidade fisiológica tem sido pesquisada com certa intensidade em várias espécies, porém é pouco freqüente em espécies florestais. A separação das sementes por classes de tamanho para determinação da qualidade fisiológica, através de testes de germinação e vigor, tem sido bastante empregada, visando encontrar a classe ideal para multiplicação das diversas espécies vegetais (Torres, 1994).

Quanto ao meio de cultivo, diversos materiais orgânicos e inorgânicos têm sido utilizados na formulação de substratos para a produção de mudas, havendo necessidade de se determinar os mais apropriados para cada espécie, de forma a atender sua demanda quanto a fornecimento de nutrientes e propriedades físicas como retenção de água, aeração, facilidade para penetração de raízes e não ser favorável à incidência de doenças (Lima et al., 2006). Maior ênfase tem sido dada à pesquisa de diferentes combinações de substratos, que claramente influenciam na germinação e vigor das sementes, bem como no desenvolvimento e a sanidade das mudas produzidas. Não obstante o mérito desses trabalhos, não se pode esquecer de que tais substratos devem ser acessíveis aos produtores rurais que, em geral, constituem um público de baixa renda.

O ambiente de cultivo ou produção de mudas pode afetar positiva ou negativamente a qualidade das plantas. Conforme Brissette et al. (1991), o sombreamento, proporcionado pelo emprego de casas de vegetação, pode ser utilizado para auxiliar no controle excessivo de temperatura, destacando que a redução da radiação solar, com telas, pode diminuir a temperatura do ambiente em até cinco graus Celsius, entre outros benefícios. Porém, de acordo com Sentelhas e Santos (1995), nem todas as alterações são benéficas.

Tendo em vista que grande maioria das espécies de palmeiras é propagada por sementes, em alguns países existem empresas que já comercializam esse material. No Brasil, apesar dessa atividade ainda ser muito restrita, existe um grande potencial de expansão. Portanto, técnicas apropriadas para a avaliação da qualidade fisiológica e vigor de sementes devem ser desenvolvidas. Desse modo, faz-se necessário descobrir quais são as condições de ambientes, substratos e tamanhos de sementes ideais para germinação de sementes de palmeiras, que permitirão a comparação entre lotes.

O experimento foi conduzido com o objetivo de avaliar o efeito do tamanho da semente, de substratos e do ambiente na emergência de plântulas de C. hospita.

\section{MATERIAL E MÉTODOS}

A pesquisa foi desenvolvida em casa de vegetação anexa ao Laboratório de Análises de Sementes e no Setor de Horticultura do Departamento de Fitotecnia da Universidade Federal do Ceará-UFC. As sementes foram extraídas de frutos de C. hospita colhidos em janeiro de 2007, no estádio 
visual de maturação, provenientes de 15 árvores matrizes situadas na Fazenda Raposo, em Maracanaú-CE.

As sementes foram classificadas, com base no diâmetro, em quatro tamanhos: pequena $(7,7-8,95 \mathrm{~mm})$, média $(8,96-$ $10,21 \mathrm{~mm})$, grande $(10,22-11,48 \mathrm{~mm})$ e mistura, constituída de sementes retiradas aleatoriamente do lote inicial. Em seguida, estas foram semeadas em sacos de polietileno de $40 \times 40 \mathrm{~cm}$, com 20 sementes cada, contendo dois substratos distintos: substrato 1 - solo de aluvião + arisco + composto orgânico Polefértil $^{\circledR}$ (2:2:1 em volume) e substrato 2 - areia vermelha + bagana de carnaúba + húmus de minhoca (2,5:2,5:1 em volume). As Tabelas 1 e 2 apresentam, respectivamente, as análises físico-químicas dos substratos mencionados.

TABELA 1. Características físico-químicas do substrato 1 utilizado para a emergência e desenvolvimento de plântulas de C. hospita. Fortaleza-CE, 2007.

\begin{tabular}{|c|c|c|c|c|c|c|c|c|c|c|c|}
\hline \multicolumn{5}{|c|}{ Composição Granulométrica (g/kg) } & \multirow{2}{*}{\multicolumn{2}{|c|}{$\begin{array}{c}\text { Classificação } \\
\text { Textural }\end{array}$}} & \multirow{2}{*}{\multicolumn{2}{|c|}{$\begin{array}{l}\text { Grau de } \\
\text { Floculação } \\
\text { (g/100g) }\end{array}$}} & \multicolumn{3}{|c|}{ Densidade $\left(\mathrm{g} / \mathrm{cm}^{3}\right)$} \\
\hline \multirow{2}{*}{$\begin{array}{c}\text { Areia } \\
\text { Grossa }\end{array}$} & \multirow{2}{*}{$\begin{array}{c}\begin{array}{c}\text { Areia } \\
\text { Fina }\end{array} \\
230\end{array}$} & \multirow{2}{*}{$\begin{array}{c}\text { Silte } \\
60\end{array}$} & \multirow{2}{*}{$\frac{\text { Argila }}{70}$} & \multirow{2}{*}{$\begin{array}{c}\begin{array}{c}\text { Argila } \\
\text { Dispersa }\end{array} \\
10\end{array}$} & & & & & \multirow{2}{*}{\multicolumn{2}{|c|}{$\begin{array}{c}\text { Global } \\
1,56 \\
\end{array}$}} & \multirow{2}{*}{$\begin{array}{c}\text { Partícula } \\
2,61\end{array}$} \\
\hline & & & & & Areia fr & Inca & & & & & \\
\hline \multicolumn{3}{|c|}{ Umidade $(\mathrm{g} / 100 \mathrm{~g})$} & \multirow[b]{2}{*}{$\begin{array}{c}\mathrm{pH} \\
\text { (água) }\end{array}$} & \multirow[b]{2}{*}{ C.E $(\mathrm{dS} / \mathrm{m})$} & \multicolumn{7}{|c|}{ Complexo Sortivo $\left(\mathrm{cmol}_{\mathrm{c}} / \mathrm{kg}\right)$} \\
\hline $\begin{array}{l}0,033 \\
\mathrm{MPa}\end{array}$ & $\begin{array}{c}1,5 \\
\mathrm{MPa}\end{array}$ & $\begin{array}{c}\text { Água } \\
\text { Disponível }\end{array}$ & & & $\mathrm{Ca}^{2+}$ & $\mathrm{Mg}^{2+}$ & $\mathrm{Na}^{+}$ & $\mathrm{K}^{+}$ & $\mathrm{H}^{+}$ & $S$ & $\mathrm{~T}$ \\
\hline 3,93 & 3,41 & 0,52 & 6,5 & 2,99 & 3,50 & 1,90 & 0,52 & 1,79 & 2,81 & 7,7 & 10,5 \\
\hline \multirow{2}{*}{$\mathrm{V}(\%)$} & \multirow{2}{*}{$\begin{array}{l}\text { PST } \\
(\%)\end{array}$} & \multirow{2}{*}{$\mathrm{C}(\mathrm{g} / \mathrm{kg})$} & \multirow{2}{*}{$\begin{array}{c}\mathrm{N} \\
(\mathrm{g} / \mathrm{kg})\end{array}$} & \multirow{2}{*}{$\mathrm{C} / \mathrm{N}$} & M.O & \multirow{2}{*}{\multicolumn{2}{|c|}{$\begin{array}{c}\text { P assimilável } \\
(\mathrm{mg} / \mathrm{kg})\end{array}$}} & \multicolumn{4}{|c|}{ Micronutrientes (ppm) } \\
\hline & & & & & $(\mathrm{g} / \mathrm{kg})$ & & & $\mathrm{Fe}$ & $\mathrm{Cu}$ & $\mathrm{Zn}$ & $\mathrm{Mn}$ \\
\hline 73 & 5 & 6,96 & 0,71 & 10 & 12,00 & & & 42,5 & 0,8 & 5,9 & 12,4 \\
\hline
\end{tabular}

$(\mathrm{pH})$ potencial hidrogeniônico, (C.E) condutividade elétrica, (S) soma de bases, (T) capacidade de troca de cátions total a pH 7,0, (V) saturação por bases, (PST) percentagem de sódio trocável, (M.O) matéria orgânica.

TABELA 2. Características físico-químicas do substrato 2 utilizado para a emergência e desenvolvimento de plântulas de C. hospita. Fortaleza-CE, 2007.

\begin{tabular}{|c|c|c|c|c|c|c|c|c|c|c|c|}
\hline \multicolumn{5}{|c|}{ Composição Granulométrica (g/kg) } & \multirow{2}{*}{\multicolumn{2}{|c|}{$\begin{array}{c}\text { Classificação } \\
\text { Textural }\end{array}$}} & \multirow{2}{*}{\multicolumn{2}{|c|}{$\begin{array}{l}\text { Grau de } \\
\text { Floculação } \\
(\mathrm{g} / 100 \mathrm{~g})\end{array}$}} & \multicolumn{3}{|c|}{ Densidade $\left(\mathrm{g} / \mathrm{cm}^{3}\right)$} \\
\hline \multirow{2}{*}{$\begin{array}{c}\begin{array}{c}\text { Areia } \\
\text { Grossa }\end{array} \\
580\end{array}$} & \multirow{2}{*}{$\begin{array}{c}\text { Areia } \\
\text { Fina }\end{array}$} & \multirow{2}{*}{$\begin{array}{c}\text { Silte } \\
100\end{array}$} & \multirow{2}{*}{$\begin{array}{c}\text { Argila } \\
60\end{array}$} & \multirow{2}{*}{$\begin{array}{c}\begin{array}{c}\text { Argila } \\
\text { Dispersa }\end{array} \\
50\end{array}$} & & & & & \multirow{2}{*}{\multicolumn{2}{|c|}{$\begin{array}{c}\text { Global } \\
1,30\end{array}$}} & \multirow{2}{*}{$\begin{array}{c}\text { Partícula } \\
2,45\end{array}$} \\
\hline & & & & & Areia 1 & anca & & & & & \\
\hline \multicolumn{3}{|c|}{ Umidade $(\mathrm{g} / 100 \mathrm{~g})$} & \multirow{2}{*}{$\begin{array}{c}\mathrm{pH} \\
\text { (água) }\end{array}$} & \multirow{2}{*}{$\begin{array}{c}\text { C.E } \\
(\mathrm{dS} / \mathrm{m})\end{array}$} & \multicolumn{7}{|c|}{ Complexo Sortivo $\left(\mathrm{cmol}_{\mathrm{c}} / \mathrm{kg}\right)$} \\
\hline $\begin{array}{l}0,033 \\
\mathrm{MPa}\end{array}$ & $\begin{array}{c}1,5 \\
\mathrm{MPa}\end{array}$ & $\begin{array}{c}\text { Água } \\
\text { Disponível }\end{array}$ & & & $\mathrm{Ca}^{2+}$ & $\mathrm{Mg}^{2+}$ & $\mathrm{Na}^{+}$ & $\mathrm{K}^{+}$ & $\mathrm{H}^{+}$ & $S$ & $\mathrm{~T}$ \\
\hline 11,27 & 11,13 & 0,15 & 6,7 & 7,53 & 8,50 & 4,00 & 1,46 & 4,99 & 2,81 & 18,9 & 21,8 \\
\hline \multirow{2}{*}{$\mathrm{V}(\%)$} & \multirow{2}{*}{$\begin{array}{l}\text { PST } \\
(\%)\end{array}$} & \multirow{2}{*}{$\mathrm{C}(\mathrm{g} / \mathrm{kg})$} & \multirow{2}{*}{$\begin{array}{c}\mathrm{N} \\
(\mathrm{g} / \mathrm{kg})\end{array}$} & \multirow{2}{*}{$\mathrm{C} / \mathrm{N}$} & \multirow{2}{*}{$\begin{array}{l}\text { M.O } \\
(\mathrm{g} / \mathrm{kg})\end{array}$} & \multirow{2}{*}{\multicolumn{2}{|c|}{$\begin{array}{c}\text { P assimilável } \\
(\mathrm{mg} / \mathrm{kg})\end{array}$}} & \multicolumn{4}{|c|}{ Micronutrientes (ppm) } \\
\hline & & & & & & & & $\mathrm{Fe}$ & $\mathrm{Cu}$ & $\mathrm{Zn}$ & $\mathrm{Mn}$ \\
\hline 87 & 7 & 25,8 & 1,30 & 10 & 44,48 & & & 42,46 & 0,80 & 5,85 & 12,37 \\
\hline
\end{tabular}

(pH) potencial hidrogeniônico, (C.E) condutividade elétrica, (S) soma de bases, (T) capacidade de troca de cátions total a pH 7,0, (V) saturação por bases, (PST) percentagem de sódio trocável, (M.O) matéria orgânica.

Os recipientes foram mantidos em casa de vegetação e ambiente a pleno sol até o final do experimento. A temperatura e a umidade relativa do ar, em função das horas do dia, nos dois ambientes, foram medidas com o auxílio de um higrotermômetro HT-210 SAMMAR $^{\circledR}$, cujos valores médios obtidos no decorrer do ensaio são apresentados na Figura 1. 

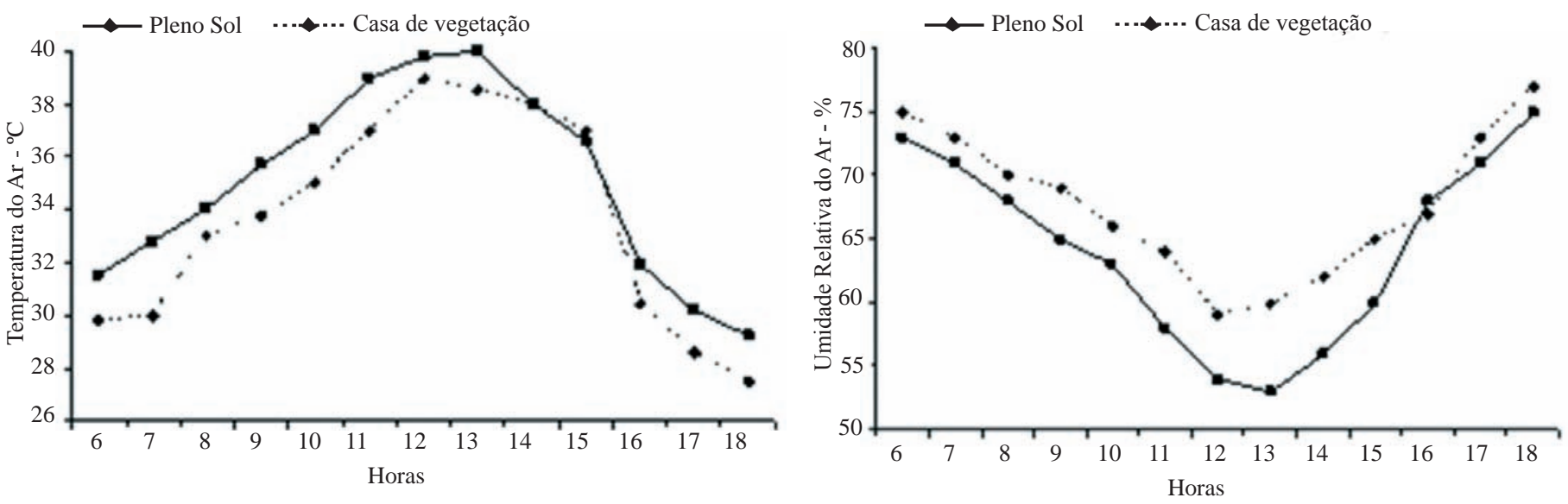

FIGURA 1. Valores médios de temperatura e umidade relativa do ar ao longo do dia, nos ambientes de cultivo de $C$. hospita. Fortaleza-CE, 2007.

Na emergência considerou-se como plântula normal àquela com capacidade de continuar o seu desenvolvimento em campo. A emergência foi monitorada diariamente, até o $60^{\circ}$ dia após a semeadura, para o cálculo do índice de velocidade de emergência (Maguire, 1962) e tempo médio de emergência (Labouriau, 1983).

Adotou-se o delineamento experimental inteiramente casualizado, com quatro repetições de 20 sementes, em esquema fatorial $4 \times 2 \times 2$ (quatro classes de tamanhos de sementes; dois substratos e dois ambientes), totalizando

Substrato $1 \quad \boldsymbol{\Delta}$ Substrato 2

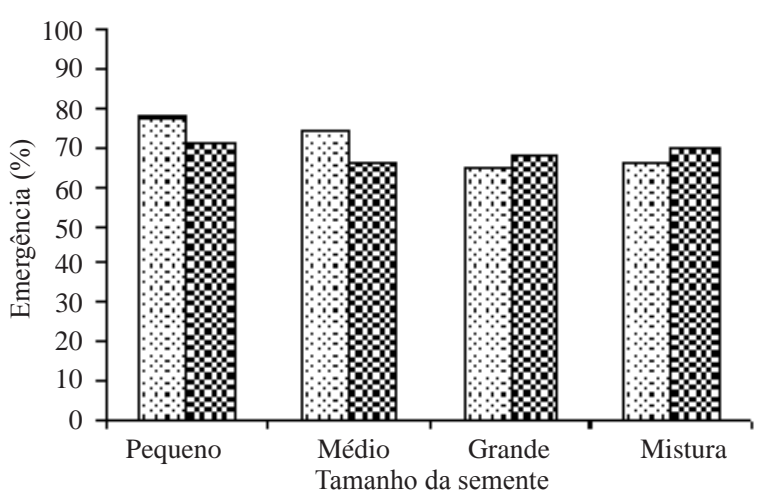

16 tratamentos. A comparação das médias foi realizada por intermédio do teste de Tukey, em nível de 5\% de probabilidade (Banzatto e Kronka, 2006).

\section{RESULTADOS E DISCUSSÃO}

Em relação ao percentual de emergência de plântulas, não foi observada diferença significativa entre os tratamentos, revelando que, para a espécie C. hospita, os fatores avaliados não exerceram influência sobre esta variável (Figura 2).

FIGURA 2. Emergência de plântulas de $C$. hospita oriundas de quatro tamanhos de sementes e cultivadas em dois tipos de substratos e duas condições ambientais. Fortaleza-CE, 2007.

De acordo com Cazetta et al (1995), nem sempre pode ser constatada interferência do tamanho da semente sobre a emergência. Quanto ao substrato, os dados obtidos neste trabalho são conciliáveis aos de Iossi et al. (2003), que também não observaram efeito deste fator sobre a emergência de plântulas de tamareira-anã (Phoenix roebelenii O’ Brien), uma palmeira ornamental. No que diz respeito aos ambientes, apesar de ter ocorrido uma variação de aproximadamente 17 pontos percentuais entre os valores médios de emergência de plântulas (64 e 81\%), não foi observada diferença estatística significativa entre os tratamentos. Logo as plântulas da espécie em questão emergem tanto em ambientes sombreados, quanto 
em pleno sol. Labouriau (1983) menciona que a distribuição geográfica e as preferências ecológicas de muitas espécies são determinadas pela faixa de condições ambientais toleradas pela germinação de suas sementes.

O substrato constituído de areia vermelha, bagana de carnaúba e húmus de minhoca $(2,5: 2,5: 1)$, combinado com o ambiente pleno sol, foi o tratamento que proporcionou o menor valor médio para o índice de velocidade de emergência $(0,37)$, diferindo significativamente dos demais (Figura 3). Portanto, caso a espécie em questão venha a ser cultivada a pleno sol, o substrato utilizado deverá possuir as mesmas características do substrato 1 (Tabela 1), a fim de se obter uma emergência mais rápida. Germinação rápida e uniforme das sementes, seguida por imediata emergência das plântulas são características altamente desejáveis na produção de mudas, pois de acordo com Martins et al. (1999), quanto mais tempo a plântula demorar a emergir do solo, mais susceptível estará às condições ambientais.

Pleno Sol

B Casa de vegetação

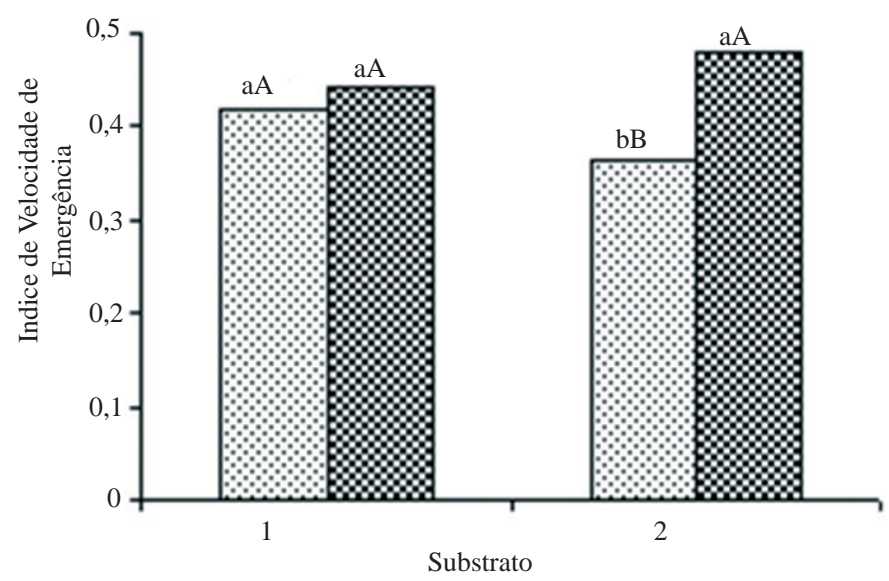

FIGURA 3. Índice de velocidade de emergência de plântulas de $C$. hospita cultivadas em dois tipos de substratos e duas condições ambientais. Fortaleza-CE, 2007.

Letras minúsculas comparam os ambientes em cada substrato e letras maiúsculas comparam os substratos em cada ambiente.

A velocidade de emergência de plântulas de $C$. hospita não foi influenciada pelo tamanho da semente, cujas sementes grandes, embora contendo aparentemente maior quantidade de reservas, não apresentaram o melhor desempenho germinativo. Resultados similares foram encontrados por Ferreira e Torres (2000) estudando a influência do tamanho das sementes de Acacia senegal (L.) Willd. na germinação e no vigor, os quais verificaram que a porcentagem e o índice de velocidade de emergência não foram afetados pelo tamanho das sementes. Andrade e Paulino (1995), conduzindo estudos com palmiteiro (Euterpe edulis Martius), obtiveram resultados semelhantes, pois o tamanho da semente também não influenciou na velocidade de emergência desta espécie. Por outro lado, Ledo et al. (2002) obtiveram maiores índices para esta variável quando utilizaram sementes de pupunha de tamanho médio e grande, diferindo estatisticamente das sementes pequenas.

O cultivo de $C$. hospita utilizando sementes sem padronização de tamanho, ou seja, o tratamento mistura, assim como sementes de tamanho pequeno, proporcionaram os menores tempos de emergência, quais sejam 33 e 34 dias, respectivamente (Figura 4). Deste modo, nestes tratamentos foi obtida uma emergência mais rápida, a qual pode beneficiar, não só o vigor da plântula, como a formação da muda.
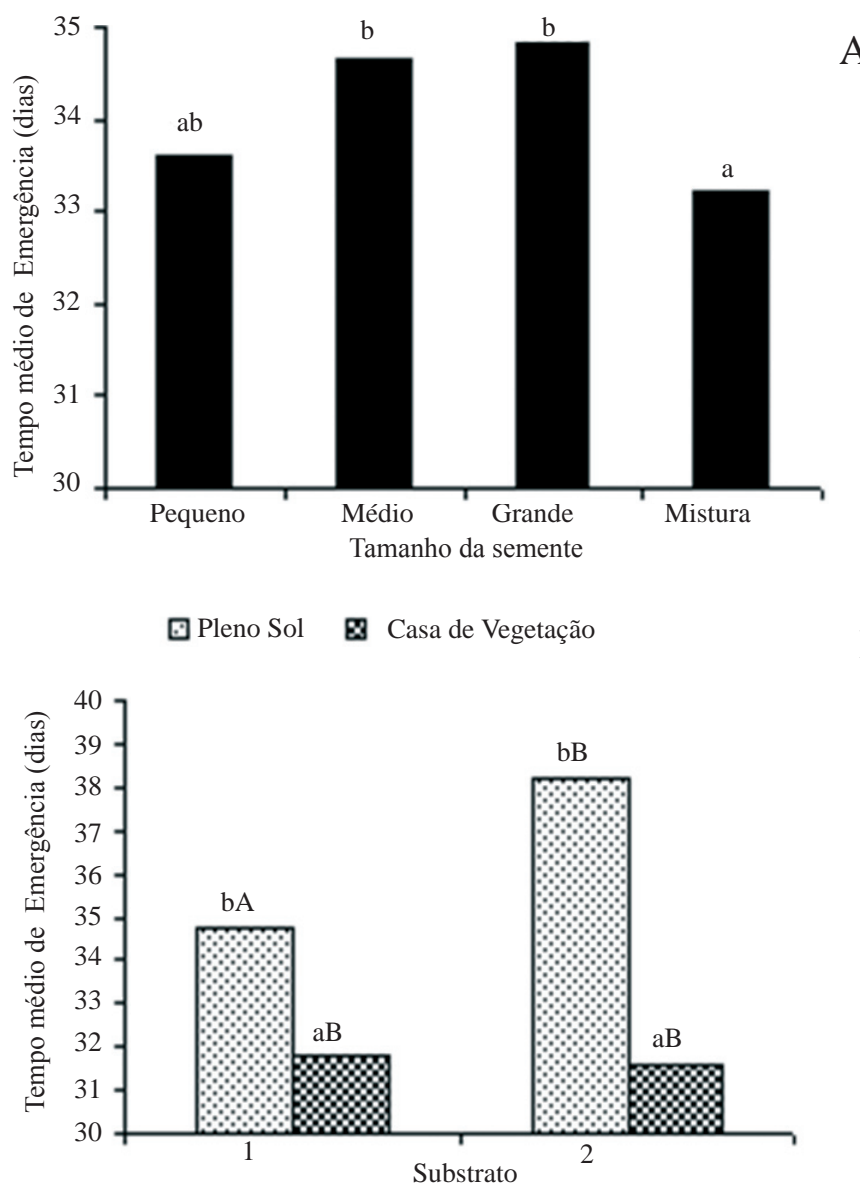

FIGURA 4. Tempo médio de emergência de plântulas de $C$. hospita oriundas de quatro tamanhos de sementes e cultivadas em dois tipos de substratos e duas condições ambientais. Fortaleza-CE, 2007.

Letras minúsculas comparam os ambientes em cada substrato e letras maiúsculas comparam os substratos em cada ambiente. 
Nos testes que avaliaram características das plântulas, as sementes menores, por necessitarem de menor quantidade de água, foram as primeiras a germinar (Krzyzanowski et al., 1999). Em palmito-vermelho (Euterpe espiritosantensis Fernandes), a germinação uniforme das sementes foi uma característica importante na formação de mudas, pois, quanto mais tempo a plântula levou para emergir na superfície do solo e permaneceu nos estádios iniciais de desenvolvimento, mais vulnerável esteve nas condições do meio (Martins et al., 2000).

A interação entre o substrato e o ambiente mostrou que no cultivo a pleno sol, o substrato constituído de solo de aluvião, arisco e composto orgânico (2:2:1), possibilitou uma emergência mais rápida que a mistura de areia vermelha, bagana de carnaúba e húmus de minhoca (2,5:2,5:1), apresentando emergência de plântulas por volta de 35 dias após a semeadura, três dias a menos do que este último (Figura 4).

Ossubstratospossuemdiferentesníveis dedisponibilidade de água de acordo com suas características físicas (Figliolia e Pina-Rodrigues, 1995). Desta forma, essas características podem ter influenciado a velocidade da embebição e, por conseguinte, o tempo médio de emergência. Além disso, o ambiente sombreado pode ter reduzido os efeitos maléficos das altas temperaturas (Figura 1).

No que diz respeito aos ambientes de cultivo, a casa de vegetação se destacou em relação ao ambiente não sombreado, com tempo médio de emergência de aproximadamente 32 dias em ambos os substratos, em detrimento dos 35 e 38 dias necessários para emergência de plântulas cultivadas nos substratos 1 e 2, respectivamente, a pleno sol. Este resultado pode estar relacionado com as menores temperaturas e maiores umidades relativas do ar registradas ao longo do dia no ambiente protegido (Figura 1).

A temperatura ideal para germinação de sementes, segundo Ramos e Varela (2003), geralmente varia dentro da faixa de temperatura encontrada no local e na época ideal para a emergência e estabelecimento das plântulas. Carpenter (1988) estudou os limites de temperatura para a germinação de sementes de quatro espécies de palmeiras: Acoelorraphe wrightii, Coccothrinax argentata, Sabal etonia e Thrinax morrisii. A germinação foi melhor a $35^{\circ} \mathrm{C}$, sendo que temperaturas de 5 a $10^{\circ} \mathrm{C}$ acima ou abaixo de $35^{\circ} \mathrm{C}$, freqüentemente, retardaram e reduziram a germinação, e tornando-a irregular e desuniforme. De acordo com Lorenzi et al. (2004), a germinação de sementes de várias espécies de palmeiras é favorecida por temperaturas entre $24^{\circ} \mathrm{C}$ e $28^{\circ} \mathrm{C}$ e umidade relativa do ar de aproximadamente $70 \%$.
No entanto, Iossi et al. (2003) comentam que as condições de temperatura e substratos ideais para a germinação de sementes de palmeiras são variáveis, pelo menos entre os gêneros. Variações no período germinativo entre genótipos de Euterpe edulis Martius foram associadas a uma estratégia adaptativa, possibilitando a entrada gradual de indivíduos no banco de plântulas (Reis et al., 1992).

\section{CONCLUSÕES}

Nas condições de realização do experimento conclui-se que: o tamanho da semente, o tipo de substrato e o ambiente não influenciam no percentual de emergência de plântulas.

Sementes de Copernicia hospita oriundas de mistura e de tamanho pequeno, semeadas em ambos os substratos solo de aluvião + arisco + composto orgânico Polefértil ${ }^{\circledR}$ (2:2:1 em volume) e areia vermelha + bagana de carnaúba + húmus de minhoca (2,5:2,5:1 em volume) em condições de casa de vegetação, proporcionam emergência mais rápida das plântulas.

\section{REFERÊNCIAS}

ANDRADE, A.C.S.; PAULINO, M.T.S. Efeito da massa da semente na velocidade de germinação e no desenvolvimento deplântulas deEuterpeedulis Mart. (palmiteiro). Informativo Abrates, Londrina, v.5, n.2, p.189, 1995.

BANZATTO, D.A.; KRONKA, S.N. Experimentação agrícola. 4.ed. Jaboticabal: UNESP. 2006. 237p.

BRISSETTE, J.C.; BARNETT, T.J.; LANDIS, T.D. Container Seedlings. In: DURYEA, M.L., DOUGHERTY, P.M. (Eds) Forest regeneration manual, Dordrecht: Kluwer Academic Publishers, 1991. p.117-41.

BROSCHAT, T.K. Palm seed propagation. Acta Horticulturae, Wageningen, n.360, p.141-147, 1994.

BROSCHAT, T.K.; MEEROW, A. W.; Ornamental Palm Horticulture. 1ed., Gainesville: University Press of Florida, 2000. 255p.

CARPENTER, W.J. Temperature affects seed germination of four Florida palm species. HortiScience, Alexandria, v.23, p.336-337, 1988.

CAZETTA, J.O.; SADER, R.; IKEDA, M. Efeito do tamanho no desempenho germinativo de sementes de feijoeiro (Phaseolus vulgaris L.). Revista Científica, São Paulo, v.23, n.1, p.65-71, 1995.

FERREIRA, M.G.R.; TORRES, S.B. Influência do tamanho 
das sementes na germinação e no vigor de plântulas de Acacia Senegal (L.) Willd. Revista Brasileira de Sementes, Brasília, v.22, n.1, p.271-275, 2000.

FIGLIOLIA, M.B.; PIÑA-RODRIGUES, F.C.M. Considerações práticas sobre testes de germinação. In: SILVA, A.; PIÑA-RODRIGUES, F.C.M.; FIGLIOLIA, M.B. Manual Técnico de Sementes Florestais. São Paulo: Instituto Florestal, 1995. p.1-12. (Série Registros, 14).

KRZYZANOWSKI, F.C.; VIEIRA, R.D.; FRANÇA-NETO, J.B. Vigor de sementes: conceitos e testes. Londrina: ABRATES, 1999. 218p.

IOSSI, E.; SADER, R.; PIVETTA, K.F.L.; BARBOSA, J.C. Efeitos de substratos e temperaturas na germinação de sementes de tamareira-anã (Phoenix roebelenii O' Brien). Revista Brasileira de Sementes, Brasília, v.25, n.2, p.6369, 2003.

LABOURIAU, L.G. A germinação das sementes. Washington: Secretaria geral da Organização dos Estados Americanos, 1983. 174p.

LEDO, A. da S.; MEDEIROS-FILHO, S.; LEDO, F.J. da S.; ARAÚJO, E.C. Efeito do tamanho da semente, do substrato e pré-tratamento na germinação de sementes de pupunha. Revista Ciência Agronômica, Fortaleza, v.33, n.1, p.29-32, 2002.

LIMA, R.L.S. SEVERINO,L.S.; SILVA, M.I.L.;VALE, L.S.; BELTRÃO, N.E.M. Volume de recipientes e composição de substratos para produção de mudas de mamoneira. Revista Ciência e Agrotecnologia, Lavras, v.30, n.3, p.480-486, 2006.
LORENZI, H.; SOUZA, H. M. de; MEDEIROS-COSTA, J. T. de.; CERQUEIRA, L. S. C. de; FERREIRA, E. J. L. Palmeiras Brasileiras e Exóticas Cultivadas. Nova Odessa: Plantarum, 2004. 416p.

MAGUIRE, J.D. Speed of germination aid in selection and evaluation for seedling emergence and vigor. Crop Science, Madison, v.2, n.1, p.176-177. 1962.

MARTINS, C.C.; NAKAGAWA, J.; BOVI, M.L.A. Efeito da posição da semente no substrato e no crescimento inicial das plântulas de Palmito-Vermelho (Euterpe espiritosantensis Fernandes - Palmae). Revista Brasileira de Sementes, Brasília, v.21, n.1, p.164-173, 1999.

MARTINS, C.C.; NAKAGAWA, J.; BOVI, M.L.A.; STANGUERLIM, H. Influência do peso das sementes de palmito-vermelho (Euterpe espiritosantensis Fernandes) na porcentagem e na velocidade de germinação. Revista Brasileira de Sementes, Brasília, v.22, n.1, p.47-53, 2000.

RAMOS, M.B.P.; VARELA, V.P. Efeito da temperatura e do substrato sobre a germinação de sementes de visgueiro do igapó (Parkia discolor Benth) LEGUMINOSAE, MIMOSOIDEAE. Revista de Ciências Agrárias, Belém, n.39, p.135-143, 2003.

REIS, M.S.; FRANCHINI, R.G.; FANTINI, A.C. Variação no período germinativo em sementes de Euterpe edulis Martius procedentes da região de Morretes-PR. Revista do Instituto Florestal, São Paulo, v.4, p.1252-1255, 1992.

TORRES, S.B. Influência do tamanho das sementes de Acacia gomifera no desenvolvimento das mudas. Agropecuária Catarinense, Santa Catarina, v.7, n.2, p.5, 1994. 